\title{
Arbitrary control of coherent dynamics for distant qubits in a quantum network
}

\author{
Shi-Biao Zheng ${ }^{1,2}$, Chui-Ping Yang $^{1,3,4}$, and Franco Nori ${ }^{1,4}$ \\ ${ }^{1}$ Advanced Science Institute, Riken, \\ Wako-shi, Saitama 351-0198, Japan \\ ${ }^{2}$ Department of Physics, Fuzhou University, Fuzhou 350002, China \\ ${ }^{3}$ Department of Physics, Hangzhou Normal University, \\ Hangzhou, Zhejiang 310036, China and \\ ${ }^{4}$ Department of Physics, University of Michigan, Ann Arbor, MI 48109, USA
}

(Dated: August 20, 2018)

\begin{abstract}
We show that the coherent coupling of atomic qubits at distant nodes of a quantum network, composed of several cavities linked by optical fibers, can be arbitrarily controlled via the selective pairing of Raman transitions. The adiabatic elimination of the atomic excited states and photonic states leads to selective qubit-qubit interactions, which would have important applications in quantum information processing. Quantum gates between any pair of distant qubits and parallel two-qubit operations on selected qubit pairs can be implemented through suitable choices of the parameters of the external fields. The selective pairing of Raman transitions also allows the generation of spin chains and cluster states without the requirement that the cavity-fiber coupling be smaller than the detunings of the Raman transitions.
\end{abstract}

PACS numbers: 03.67.Mn 


\section{INTRODUCTION}

Controlling the coherent dynamics of many-qubit systems lies at the heart of quantum information. In the standard model of a quantum computer, quantum information is stored in a quantum register composed of many qubits. The performance of a certain quantum computational task corresponds to the control of the unitary coherent evolution of the qubits. Two-qubit quantum phase gates and multi-qubit entanglement have been achieved experimentally in various systems. For example, cavity QED with atoms trapped in an optical cavity can couple atomic qubits via photons (see, e.g., [1]). However, the practical implementation of quantum computing requires a large number of qubits, which is extremely difficult to achieve experimentally in a single cavity. This is due to the fact that the spatial separation between neighboring qubits decreases as the number of qubits increases, and thus individual addressing becomes increasingly difficult.

The coherent coupling of separate qubits is of importance for implementing deterministic long-distance entanglement and large-scale quantum information processing. The entanglement of distant qubits is an essential ingredient for testing quantum nonlocality against local-hidden-variable theories [2,3] and a key resource for quantum communication $[4,5]$. Furthermore, quantum logic operations between distant qubits at separate nodes in a network are a prerequisite for linking several spatially-separated quantum registers to build a quantum computer. Moreover, the next-nearest-neighbor interaction in a spin- $1 / 2$ chain can be useful for producing cluster states (see, e.g., [6,7]), which is the resource for one-way quantum computation.

Recently, schemes have been proposed [8-10] to realize quantum communication, deterministic entanglement, and phase gates between two atomic qubits trapped in separate optical cavities, which are coupled by an optical fiber via coherent dynamics. These previous works [8-10] concentrate on the simplest case: the system is only composed of two nodes. In order to implement a distributed quantum computational network with several nodes, it is necessary to be able to control the coupling of different nodes, exploit suitable coupling dynamics to perform desired logic operations between any pair of nodes, and engineer entanglement among these nodes. Unfortunately, these issues have not been addressed yet.

The controlled dynamics of strongly-interacting many-particle systems is also of importance in studying quantum phase transitions, which involves complex collective quantum mechanical behavior. Also, much attention has been devoted to the ground state entanglement in spin chains near and at the critical point [11-13], which is responsible for long-range correlations. Recently, Hartmann et al. [14] have shown that effective spin lattices can be produced with atoms trapped in an array of microcavities. The off-resonant Raman transitions between two ground states, induced by the cavity modes and external fields, lead to spin-spin coupling. In order to generate a spin chain, in which each qubit is only coupled to its nearest neighbors, the tunneling rate of photons between neighboring cavities should be much smaller than the detunings of the Raman transitions so that the nearest-neighbor coupling dominates the dynamics. Meanwhile, the photon tunneling rate should be much larger than the cavity decay rate. It is extremely hard to simultaneously satisfy these two requirements in experiments.

Here we theoretically show that one can arbitrarily control the coherent coupling dynamics of multiple atomic qubits at distant nodes of a quantum network, which is composed of several cavities linked by optical fibers. This is based on the pairing of off-resonant Raman transitions, through which the Raman transitions of each qubit can only be coupled to those of selected qubits to produce the desired qubit-qubit interaction. We present two applica- 


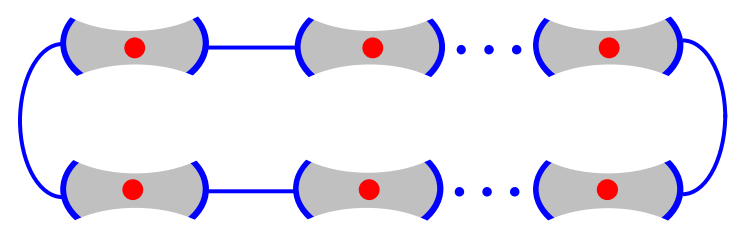

FIG. 1: (color online) Schematic diagram of $n$ distant atoms trapped in separate coupled cavities, which are connected by short optical fibers.

tions of this physical mechanism. First, we show that gate operations between any pair of atomic qubits and selective parallel two-qubit operations on different qubit pairs can be implemented in the quantum network without exciting both the atoms and field modes, which could be useful towards future scalable quantum computing networks. Second, we show that various spin- $1 / 2$ chains can be constructed. As the cavity-fiber coupling does not need to be smaller than the detunings of the Raman transitions, much stronger spin-spin couplings can be obtained, offering the possibility for producing cluster states and observing quantum phenomena in strongly correlated quantum many-body systems, which were previously not experimentally accessible.

This paper is organized as follows. In Sec. 2, we study the coherent coupling dynamics of multiple atomic qubits trapped in separate cavities linked by optical fibers. We show that, under certain conditions, the atomic excited states and photonic states can be adiabatically eliminated, and one can pair off-resonant Raman transitions to produce controlled spin couplings. In Sec. 3, we present a scheme to implement gate operations between any pair of atomic qubits and selective parallel two-qubit operations on different qubit pairs in a quantum network based on controlled spin couplings. In Sec. 4, we show that spin chains and cluster states can be generated through pairing off-resonant Raman transitions for neighboring qubits. In Sec. 5, we address several experimental issues. Conclusions appear in Sec. 6.

\section{CONTROLLED COHERENT COUPLING DYNAMICS}

Let us consider $n$ identical atoms trapped in separated cavities connected by optical fibers, as shown in Fig. 1. In the short-fiber limit, essentially only one fiber mode interacts with the cavity modes [9]. We denote $\sum_{l} \equiv \sum_{l=1}^{n}, \sum_{m} \equiv \sum_{m=1}^{n}$, and $\sum_{k} \equiv \sum_{k=1}^{2 n}$. Then the coupling between the cavity modes and fibers are given by the interaction Hamiltonian

$$
H_{1}=\sum_{l} \nu b_{l}\left(a_{l}^{\dagger}+a_{l+1}^{\dagger}\right)+H . c .
$$

where $b_{l}$ is the annihilation operator for the $l$ th fiber mode, $a_{l}^{\dagger}$ is the creation operator for the $l$ th cavity mode, and $\nu$ is the cavity-fiber coupling strength. Here we adopt periodic boundary conditions, i.e., $b_{n+1}=b_{1}$, which can be satisfied by linking the first and the $n$th cavity using another fiber. The atomic level configuration is shown in Fig. 2(a). Each atom has one excited state $|r\rangle$ and two ground states $|e\rangle$ and $|g\rangle$. The transition $\left|e_{l}\right\rangle \rightarrow\left|r_{l}\right\rangle$ of qubit $l$ is driven by a classical laser field with Rabi frequency $\Omega_{l}$, while the transition $\left|g_{l}\right\rangle \rightarrow\left|r_{l}\right\rangle$ is coupled to the cavity mode with the coupling constant $g$. Let us assume that the classical field driving the $l$ th atom and the cavity mode are detuned from the respective transitions 


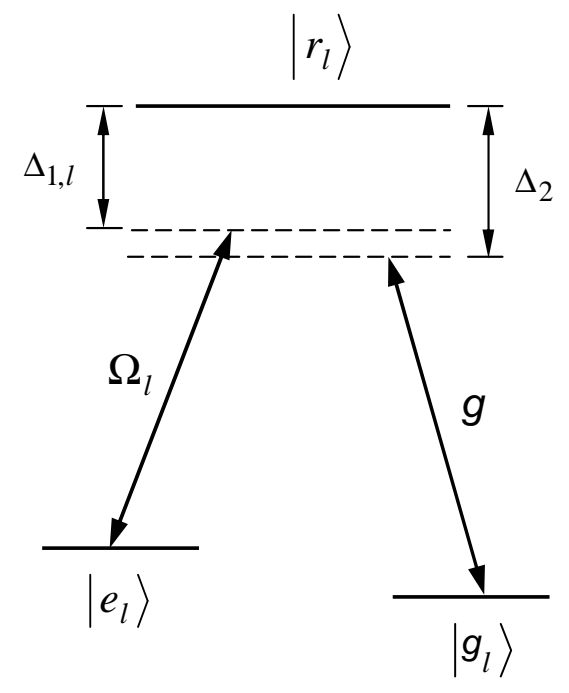

(a)

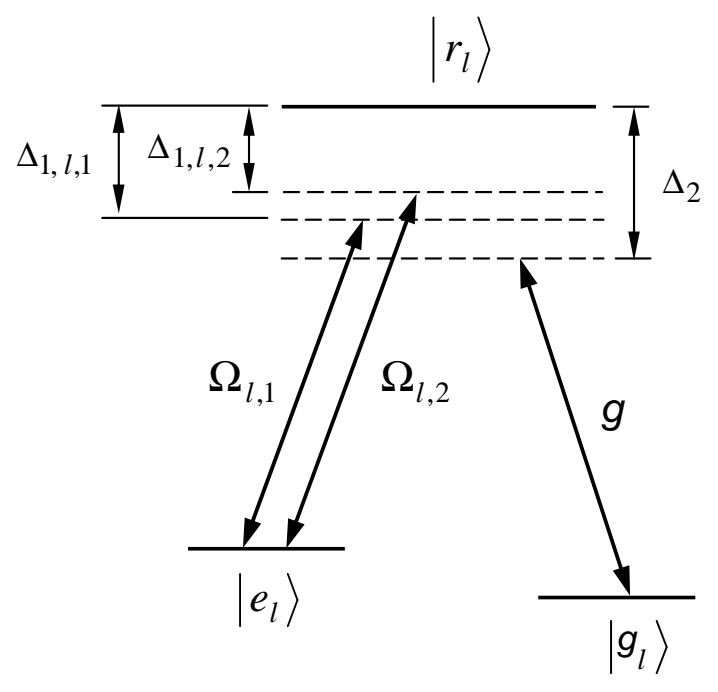

(b)

FIG. 2: (a) Atomic level configuration and transitions to implement quantum gates between any pair of qubits and parallel two-qubit operations. The transition $\left|e_{l}\right\rangle \rightarrow\left|r_{l}\right\rangle$ of the $l$ th atom is driven by a classical laser field with detuning $\Delta_{1, l}$ and Rabi frequency $\Omega_{l}$, while the transition $\left|g_{l}\right\rangle \rightarrow\left|r_{l}\right\rangle$ is coupled to the cavity mode with the coupling constant $g$ and has a detuning $\Delta_{2}$. (b) Atomic level configuration and transitions to implement the XY model. The transition $\left|e_{l}\right\rangle \rightarrow\left|r_{l}\right\rangle$ of the $l$ th atom is driven by two classical laser fields with detunings $\Delta_{1, l, 1}$ and $\Delta_{1, l, 2}$ and Rabi frequencies $\Omega_{l, 1}$ and $\Omega_{l, 2}$, while the transition $\left|g_{l}\right\rangle \rightarrow\left|r_{l}\right\rangle$ is coupled to the cavity mode with the coupling constant $g$ and has a detuning $\Delta_{2}$.

by $\Delta_{1, l}$ and $\Delta_{2}$, respectively. In the interaction picture, the Hamiltonian describing the atom-field interaction is

$$
H_{2}=\sum_{l}\left(\Omega_{l} e^{i \Delta_{1, l} t}\left|r_{l}\right\rangle\left\langle e_{l}\left|+g a_{l} e^{i \Delta_{2} t}\right| r_{l}\right\rangle\left\langle g_{l}\right|\right)+H . c . .
$$


Introducing the nonlocal bosonic modes

$$
c_{l}=\frac{1}{\sqrt{2 n}} \sum_{m}\left[e^{-i(2 m-1) l \pi / n} a_{m}+e^{-i 2 m l \pi / n} b_{m}\right]
$$

we can rewrite the Hamiltonians $H_{1}$ and $H_{2}$ as

$$
H_{1}=2 \nu \sum_{k} \cos (\pi k / n) c_{k}^{\dagger} c_{k}
$$

and

$$
H_{2}=\sum_{l}\left[\Omega_{l} e^{i \Delta_{1, l} t}\left|r_{l}\right\rangle\left\langle e_{l}\left|+g e^{i \Delta_{2} t} \sum_{k} e^{i(2 l-1) k \pi / n} c_{k}\right| r_{l}\right\rangle\left\langle g_{l}\right|\right]+H . c .
$$

Performing the transformation $\exp \left(i H_{1} t\right)$, we obtain the atom-field tunable interaction Hamiltonian

$$
H_{2}^{\prime}=\sum_{l}\left[\Omega_{l} e^{i \Delta_{1, l} t}\left|r_{l}\right\rangle\left\langle e_{l}\left|+\frac{1}{\sqrt{2 n}} g \sum_{k} e^{i(2 l-1) k \pi / n+i\left[\Delta_{2}-2 \nu \cos (\pi k / n)\right] t} c_{k}\right| r_{l}\right\rangle\left\langle g_{l}\right|\right]+\text { H.c. }
$$

Under the conditions

$$
\Delta_{1, l},\left|\Delta_{2}-2 \nu \cos (\pi k / n)\right| \gg \Omega, \frac{1}{\sqrt{2 n}} g,
$$

the upper level $\left|r_{l}\right\rangle$ in the Hamiltonian $H_{2}^{\prime}$ can be adiabatically eliminated, leading to the couplings between the two ground states

$$
\begin{aligned}
H_{\text {int }}= & -\sum_{l}\left\{\eta_{l}\left|e_{l}\right\rangle\left\langle e_{l}\right|+\sum_{k}\left[\xi_{k} c_{k}^{\dagger} c_{k}\left|g_{l}\right\rangle\left\langle g_{l}\right|\right.\right. \\
& \left.\left.+\lambda_{k, l}\left(c_{k} S_{l}^{+} e^{-i(2 l-1) k \pi / n} e^{i \delta_{k, l} t}+H . c .\right)\right]\right\}
\end{aligned}
$$

where

$$
\begin{aligned}
\eta_{l} & =\Omega_{l}^{2} / \Delta_{1, l} \\
\xi_{k} & =g^{2}\left[2 n\left(\Delta_{2}-2 \nu \cos (k \pi / n)\right]^{-1},\right. \\
\lambda_{k, l} & =\frac{\Omega_{l} g}{2 \sqrt{2 n}}\left\{\Delta_{1}^{-1}+\left[\Delta_{2}-2 \nu \cos (k \pi / n)\right]^{-1}\right\}, \\
\delta_{k, l} & =\Delta_{2}-2 \nu \cos (\pi k / n)-\Delta_{1, l}, \\
S_{l}^{+} & =\left|e_{l}\right\rangle\left\langle g_{l}\left|, S_{l}^{-}=\right| g_{l}\right\rangle\left\langle e_{l}\right| .
\end{aligned}
$$

The Hamiltonian $H_{\text {int }}$ describes multiple off-resonant Raman transitions for each atom induced by the classical field and the bosonic modes $c_{k}$. Under the condition $\delta_{k, l} \gg \lambda_{k, l}, \eta_{l}$, $\xi_{k}$, the bosonic modes do not exchange quanta with the atomic system. The off-resonant Raman coupling leads to Stark shifts and couplings between the atoms. Then the effective Hamiltonian becomes

$$
\begin{aligned}
H_{\mathrm{eff}}=-\sum_{l} & \left\{\eta_{l}\left|e_{l}\right\rangle\left\langle e_{l}\right|+\sum_{k}\left[\xi_{k} c_{k}^{\dagger} c_{k}\left|g_{l}\right\rangle\left\langle g_{l}\right|+\right.\right. \\
& \mu_{k, l}\left(c_{k}^{\dagger} c_{k}\left|g_{l}\right\rangle\left\langle g_{l}\left|-c_{k} c_{k}^{\dagger}\right| e_{l}\right\rangle\left\langle e_{l}\right|\right) \\
& \left.\left.+\sum_{m}\left(\chi_{k, l, m} S_{l}^{+} S_{m}^{-} e^{i\left(\Delta_{1, m}-\Delta_{1, l}\right) t}+H . c .\right)\right]\right\},
\end{aligned}
$$


where $l \neq m, \mu_{k, l}=\lambda_{k, l}^{2} / \delta_{k, l}$, and

$$
\chi_{k, l, m}=\frac{1}{2} \lambda_{k, l} \lambda_{k, m}\left(\delta_{k, l}^{-1}+\delta_{k, m}^{-1}\right) e^{-2 i(l-m) k \pi / n} .
$$

As the quantum number of the bosonic modes conserves during the interaction, they will remain in the vacuum state if they are initially in the vacuum state. Then the effective Hamiltonian $H_{\text {eff }}$ reduces to

$$
H_{\mathrm{eff}}=\sum_{l}\left\{\varepsilon_{l}\left|e_{l}\right\rangle\left\langle e_{l}\right|+\sum_{m}\left[\chi_{l, m} S_{l}^{+} S_{m}^{-} e^{i\left(\Delta_{1, m}-\Delta_{1, l}\right) t}+H . c .\right]\right\},
$$

where $l \neq m, \varepsilon_{l}=\sum_{k} \mu_{k, l}-\eta_{l}$, and $\chi_{l, m}=\sum_{k} \chi_{k, l, m}$. The Hamiltonian (9) has the same form as the Hamiltonian describing the coupling between quantum dots in a single cavity [15]. However, the coupling between qubits is induced by multiple nonlocal bosonic modes, while the qubit-qubit coupling in Ref. [15] was induced by a single cavity mode. Since the coupling strength $\chi_{l, m}$ and the detuning $\left(\Delta_{1, m}-\Delta_{1, l}\right)$ can be controlled via the external fields, the effective coupling Hamiltonian $H_{\text {eff }}$ can be used to realize a variety of quantum logic and entanglement operations between qubits trapped in separated cavities. We note that the Hamiltonian $H_{\text {eff }}$ can also be obtained in an array of coupled cavities without using optical fibers $[14,16]$. We now consider the case when both the classical field and cavity mode drive the transition $|g\rangle \rightarrow|r\rangle$. Under the above- mentioned large-detuning conditions, the effective Hamiltonian $H_{\text {eff }}$ is given by

$$
H_{\mathrm{eff}}=\sum_{l}\left\{\varepsilon_{l}\left|g_{l}\right\rangle\left\langle g_{l}\right|+\sum_{m}\left[\chi_{l, m}\left|g_{l}\right\rangle\left\langle g_{l}|\otimes| g_{m}\right\rangle\left\langle g_{m}\right| e^{i\left(\Delta_{1, m}-\Delta_{1, l}\right) t}+H . c .\right]\right\},
$$

where $l \neq m$.

\section{SELECTIVE GATE OPERATIONS}

Let us now set

$$
\Omega_{p}=\Omega_{q}=\Omega, \Omega_{l}=0(l \neq p, q), \Delta_{1, q}=\Delta_{1, p}
$$

Then we have

$$
\lambda_{k, l}=0(l \neq p, q), \chi_{l, m}=0(l \neq p, q \text { or } m \neq p, q), \varepsilon_{p}=\varepsilon_{q}=\varepsilon .
$$

In this case, the coupling Hamiltonian (9) reduces to

$$
H_{\mathrm{eff}}=\varepsilon\left(\left|e_{p}\right\rangle\left\langle e_{p}|+| e_{q}\right\rangle\left\langle e_{q}\right|\right)+\left(\chi_{p, q} S_{p}^{+} S_{q}^{-}+H . c .\right) .
$$

This Hamiltonian, describing the selective coupling between qubits $p$ and $q$, can be used to perform entangling operations between qubits $p$ and $q$. For example, assume that the two atoms are initially in the state $\left|e_{p}\right\rangle\left|g_{q}\right\rangle$. After an interaction time $t=\pi /\left(4 \chi_{p, q}\right)$, the two qubits evolve to the maximally-entangled state $\left(\left|e_{p}\right\rangle\left|g_{q}\right\rangle-i\left|g_{p}\right\rangle\left|e_{q}\right\rangle\right) / \sqrt{2}[17]$. The coherent dynamics also allows quantum state transfer between the two distant qubits. Suppose now that qubit $p$ is initially in a superposition of states $\left|e_{p}\right\rangle$ and $\left|g_{p}\right\rangle$, and that qubit $q$ is initially in the state $\left|g_{q}\right\rangle$. After an interaction time $t=\pi /\left(2 \chi_{p, q}\right)$, the initial state of qubit $p$ is transferred to $q$. 
Selective parallel two-qubit operations can also be implemented. As an example, suppose that one wants to perform gates on qubit pairs $(p, q)$ and $(u, v)$. Then we drive each of these qubits with a laser field. The frequencies of these classical fields are suitably adjusted so that $\Delta_{1, p}=\Delta_{1, q}, \Delta_{1, u}=\Delta_{1, v}$, and $\left|\Delta_{1, p}-\Delta_{1, u}\right| \gg\left|\chi_{\alpha, \beta}\right|(\alpha=p, q$ and $\beta=u, v)$. In this case, qubit $p$ or $q$ is decoupled to $u$ or $v$ due to the large detunings. Thus qubit $p$ is only coupled to qubit $q$, and $u$ only coupled to $v$, i.e., the Raman transitions of qubit $p(u)$ is paired with those of qubit $q(v)$. Setting $\Omega_{p}=\Omega_{q}$ and $\Omega_{u}=\Omega_{v}$ we have $\varepsilon_{p}=\varepsilon_{q}$ and $\varepsilon_{u}=\varepsilon_{v}$. The effective Hamiltonian is now given by

$$
H_{\mathrm{eff}}=\varepsilon_{p} \sum_{s=p, q}\left|e_{s}\right\rangle\left\langle e_{s}\left|+\varepsilon_{u} \sum_{\mu=u, v}\right| e_{\mu}\right\rangle\left\langle e_{\mu}\right|+\left(\chi_{p, q} S_{p}^{+} S_{q}^{-}+\chi_{u, v} S_{u}^{+} S_{v}^{-}+\text {H.c. }\right) .
$$

As the coherent coupling between qubits $p$ and $q$ is not affected by that between $u$ and $v$, entangling and swap gates on qubit pairs $(p, q)$ and $(u, v)$ can be simultaneously performed.

The effective Hamiltonian (10) allows the implementation of controlled phase gates between any pair of qubits and parallel two-qubit phase gates through a suitable choice of the Rabi frequencies and detunings of the classical fields. It should be noted that the selective parallel two-qubit operations are not restricted to the case when the selected qubit pairs undergo the same kind of gate transformations. For example, assume now that the transition $|e\rangle \leftrightarrow|r\rangle$ of each of qubits $p$ and $q$ is driven by a laser field with the detuning $\Delta_{1, p}$, while transition $|g\rangle \leftrightarrow|r\rangle$ of each of qubits $u$ and $v$ is driven by a laser field with the detuning $\Delta_{1, u}$. Under the condition that $\left|\Delta_{1, p}-\Delta_{1, u}\right|$ is much larger than the respective Raman couplings, the effective Hamiltonian becomes

$$
H_{\mathrm{eff}}=\varepsilon_{p} \sum_{s=p, q}\left|e_{s}\right\rangle\left\langle e_{s}\left|+\varepsilon_{u} \sum_{\mu=u, v}\right| e_{\mu}\right\rangle\left\langle e_{\mu}\left|+\left(\chi_{p, q} S_{p}^{+} S_{q}^{-}+H . c .\right)+2 \chi_{u, v}\right| g_{u}\right\rangle\left\langle g_{u}|\otimes| g_{v}\right\rangle\left\langle g_{v}\right| \text {. }
$$

So, in principle, one can simultaneously perform different kinds of gates on qubit pairs $(p$, $q)$ and $(u, v)$, respectively.

\section{GENERATION OF SPIN CHAINS}

We note that spin chains can also be produced with such a system. We now assume that the transition $\left|e_{l}\right\rangle \rightarrow\left|r_{l}\right\rangle$ of the $l$ th atom is driven by two classical laser fields, with detunings $\Delta_{1, l, 1}$ and $\Delta_{1, l, 2}$, and Rabi frequencies $\Omega_{l, 1}$ and $\Omega_{l, 2}$, as schematically shown in Fig. 2(b). The Hamiltonian describing the Raman couplings between the two ground states now becomes

$$
\begin{aligned}
H_{\mathrm{int}}=-\sum_{d=1,2} & \sum_{l}\left\{\eta_{l, d}\left|e_{l}\right\rangle\left\langle e_{l}\right|+\sum_{k}\left[\xi_{k} c_{k}^{+} c_{k}\left|g_{l}\right\rangle\left\langle g_{l}\right|\right.\right. \\
& \left.\left.+\left(\lambda_{k, l, d} c_{k} S_{l}^{+} e^{-i(2 l-1) k \pi / n+i \delta_{k, l, d} t}+\text { H.c. }\right)\right]\right\}
\end{aligned}
$$

where

$$
\begin{aligned}
\eta_{l, d} & =\left(\Omega_{l, d}\right)^{2} / \Delta_{1, l, d}, \\
\lambda_{k, l, d} & =\frac{\Omega_{l, 1} g}{2 \sqrt{2 n}}\left\{\Delta_{1, l, d}^{-1}+\left[\Delta_{2}-2 \nu \cos (k \pi / n)\right]^{-1}\right\}, \\
\delta_{k, l, d} & =\Delta_{2}-2 \nu \cos (2 \pi k / 2 n)-\Delta_{1, l, d} .
\end{aligned}
$$


Under the conditions $\delta_{k, l, d} \gg \lambda_{k, l, d}, \eta_{l, d}, \xi_{k, d}$, the off-resonant Raman coupling for qubit $l$ induced by the $d$ th $(d=1,2)$ classical field, and that for qubit $m$ induced by the $d^{\prime}$-th $\left(d^{\prime}=1,2\right)$ classical field lead to the two-qubit coupling with coupling strength

$$
\chi_{l, m, d, d^{\prime}}=\sum_{k} \frac{1}{2} \lambda_{k, l, d} \lambda_{k, m, d^{\prime}}\left(\delta_{k, l, d}^{-1}+\delta_{k, m, d^{\prime}}^{-1}\right) e^{-i 2(l-m) k \pi / n}
$$

and detuning

$$
\Lambda_{l, m, d, d^{\prime}}=\Delta_{1, m, d^{\prime}}-\Delta_{1, l, d}
$$

The detunings are suitably chosen so that

$$
\begin{aligned}
\Lambda_{l, l-1,1,2} & =\Lambda_{l, l+1,2,1}=0, \\
\Lambda_{l, m, 1,2} & \gg \chi_{l, m, 1,2}(m \neq l-1), \\
\Lambda_{l, m, 2,1} & \gg \chi_{l, m, 1,2}(m \neq l+1) .
\end{aligned}
$$

In this case the Raman transition of qubit $l$ induced by the first (second) classical field is only paired with that of qubit $l-1(l+1)$ induced by the second (first) classical field and thus each qubit is only resonantly-coupled to its nearest neighbors. The other two-qubit couplings can be neglected due to large detunings. Under the condition that the field modes are initially in the vacuum state, the effective Hamiltonian, obtained from Eq. (14), is now given by

$$
H_{\mathrm{eff}}=\sum_{l}\left[\varepsilon_{l}\left|e_{l}\right\rangle\left\langle e_{l}\right|+\left(\chi_{l, l+1} S_{l}^{+} S_{l+1}^{-}+H . c .\right)\right]
$$

where

$$
\begin{aligned}
\chi_{l, l+1} & =\sum_{k} e^{i 2 k \pi / n} \lambda_{k, l, 2} \lambda_{k, m, 1} \delta_{k, l, 2}^{-1}, \\
\varepsilon_{l} & =\sum_{d=1,2}\left(\sum_{k} \mu_{k, l, d}-\eta_{l, d}\right) \\
\mu_{k, l, d} & =\lambda_{k, l, d}^{2} / \delta_{k, l, d}, \eta_{j, d}=\Omega_{j, d}^{2} / \Delta_{1, j, d} .
\end{aligned}
$$

We can adjust the Rabi frequencies of the classical fields so that $\chi_{l, l+1}=\chi_{l+1, l+2}=\chi$. The energy of the level $|e\rangle$ can be made identical for all qubits by using the Stark shift of another nonresonant classical field. In this case, the effective Hamiltonian corresponds to the XY model.

We now consider the case when the classical fields and cavity mode both drive the transition $|g\rangle \rightarrow|r\rangle$. After adiabatically eliminating the upper level $|r\rangle$, we obtain the Hamiltonian

$$
\begin{aligned}
H_{\mathrm{int}}=-\sum_{d=1,2} \sum_{l}\left\{\eta_{l, d}\left|g_{l}\right\rangle\left\langle g_{l}\right|+\sum_{k=1}\left[\xi_{k} c_{k}^{\dagger} c_{k}\left|g_{l}\right\rangle\left\langle g_{l}\right|\right.\right. \\
\left.\left.+\left(\lambda_{k, l, d} c_{k}\left|g_{l}\right\rangle\left\langle g_{l}\right| e^{-i(2 l-1) k \pi / n+i \delta_{k, l, d} t}+\text { H.c. }\right)\right]\right\} .
\end{aligned}
$$

As in the XY model, the detunings of the classical fields are suitably chosen so that the second classical field driving qubit $l$ is only resonant with the first classical field driving the qubit $(l+1)$ and the two corresponding off-resonant Raman transitions are paired. Then 
the population operator $\left|g_{l}\right\rangle\left\langle g_{l}\right|$ of qubit $l$ is only coupled to those of its nearest neighbors. Through a suitable choice of the Rabi frequencies of the driving fields and tuning of the energy of the level $\left|g_{l}\right\rangle$, we can obtain from Eq. (16) the effective Hamiltonian

$$
H_{\mathrm{eff}}=\sum_{l}\left(\varepsilon \frac{1-\sigma_{z, l}}{2}+\chi \frac{1-\sigma_{z, l}}{2} \frac{1-\sigma_{z, l+1}}{2}\right),
$$

where $\sigma_{z, l}=\left|e_{l}\right\rangle\left\langle e_{l}|-| g_{l}\right\rangle\left\langle g_{l}\right|$. After an interaction time $t=\pi / \chi$, the evolution operator $e^{-i H_{\text {eff }} t}$ plus the single qubit rotation $\otimes_{l=1}^{n} e^{i \varepsilon t\left|g_{l}\right\rangle\left\langle g_{l}\right|}$ leads to the cluster state $\frac{1}{2^{n / 2}} \otimes_{l=1}^{n}\left(\left|g_{l}\right\rangle \sigma_{z, l+1}+\left|e_{l}\right\rangle\right)$, which are the resources for the one-way quantum computation $[6,7]$.

\section{DISCUSSIONS ON EXPERIMENTAL ISSUES}

We now give a brief discussion on the experimental feasibility of the proposed scheme. Set $n=3, \Omega_{1}=\Omega_{3}=\nu=g, \Omega_{2}=0, \Delta_{1,1}=\Delta_{1,3}=16 g$, and $\Delta_{2}=18.5 g$. Then we have

$$
\chi_{1,3}=\sum_{k} e^{(i 4 k \pi / n)} \frac{\lambda_{k, 1}^{2}}{\delta_{k, 1}}=8.238 \times 10^{-4} g,
$$

and the time needed to complete the entangling operation between qubits 1 and 3 is $t=$ $\pi /\left(4 \chi_{1,3}\right) \simeq 9.53 \times 10^{2} / g$. The probability that the atoms undergo a transition to the excited state due to the off-resonant interaction with the classical fields is $p_{1} \simeq \Omega_{1}^{2} / \Delta_{1,1}^{2}=3.9 \times 10^{-3}$. Meanwhile, the probability that the field modes are excited due to off-resonant Raman couplings is $p_{2} \simeq \sum_{k=1} \lambda_{k, 1}^{2} / \delta_{k, 1}^{2} \simeq 3.1 \times 10^{-3}$. Thus the effective Hamiltonian $H_{\text {eff }}$ is valid. The effective decoherence rates due to the atomic spontaneous emission and the field decay are $\gamma_{e}=p_{1} \gamma$ and $\kappa_{e}=p_{2} \kappa$, where $\gamma$ and $\kappa$ are the decay rates for the atomic excited state and the field modes, respectively. We here have assumed that the cavity modes and the fiber modes have the same decay rate. The requirement $\gamma_{e}, \kappa_{e} \ll \chi_{1,3}$ means $\gamma, \kappa \ll 0.2 g$. The parameters in the microsphere cavity QED experiment reported in Ref. [18] are: $g \simeq 2 \pi \times 20$ $\mathrm{MHz}, \gamma \simeq 2 \pi \times 2.6 \mathrm{MHz}$, and $\kappa \simeq 2 \pi \times 7 \mathrm{MHz}$. The corresponding cooperativity factor $g^{2} / 2 \gamma \kappa$ is too low for the implementation of the qubit coupling. Set $\gamma \sim \kappa \sim 3 \times 10^{-3} g$. This corresponds to a cooperativity factor $g^{2} / 2 \gamma \kappa \sim 10^{5}$, which is predicted to be available [19]. Then the effective decoherent rates are $\gamma_{e}=1.17 \times 10^{-5} \mathrm{~g}$ and $\kappa_{e}=9.3 \times 10^{-6} \mathrm{~g}$. The corresponding gate fidelity is about $F \simeq 1-\left(\gamma_{e}+\kappa_{e}\right) t \simeq 98 \%$. A near-perfect fiber-cavity coupling with an efficiency larger than $99.9 \%$ can be realized using fiber-taper coupling to high- $Q$ silica microspheres [20]. The fiber loss at $852 \mathrm{~nm}$ wavelength is about $2.2 \mathrm{~dB} / \mathrm{km}$ [21], which corresponds to the fiber decay rate $1.52 \times 10^{5} \mathrm{~Hz}$, much smaller than the available cavity decay rate. This implies that the effective decoherence rate due to the field decay should be smaller than $p_{2} \kappa$.

\section{CONCLUSIONS}

In conclusion, we have theoretically shown that the coherent coupling of multiple atoms trapped in separated cavities connected by optical fibers can be arbitrarily controlled through pairing off-resonant Raman transitions of different atoms. With this physical mechanism, quantum gates between any pair of qubits and parallel two-qubit operations in the network can be performed, and various spin chains can be generated. The cavity-fiber coupling does 
not need to be smaller than the detunings of the Raman transitions. For the same coupling to the cavity mode, the effective spin-spin coupling in our approach exceeds the previous one [14] by at least one order of magnitude, which is important for the generation of cluster states and the observation of ground-state entanglement and quantum phase transitions in quantum many-body systems. An anisotropic spin chain can be produced through pairing off-resonant balanced Raman transitions between two ground atomic states [22], in which the counter-rotating terms $c_{k} S_{l}^{+}$and $c_{k}^{\dagger} S_{l}^{+}$are involved.

\section{ACKNOWLEDGEMENTS}

We acknowledge partial support from the Laboratory of Physical Sciences, National Security Agency, Army Research Office, National Science Foundation Under Grant. No. 0726909, DARPA, JSPS-RFBR under Grant No. 09-02-92114, Grant-in-Aid for Scientific Research (S), MEXT Kakenhi on Quantum Cybernetics, and FIRST (Funding Program for Innovative R\&D on S\&T). S.B. Zheng acknowledges support from the National Natural Science Foundation of China under Grant No. 10674025, the Doctoral Foundation of the Ministry of Education of China under Grant No. 20070386002, and funds from the State Key Laboratory Breeding Base of Photocatalysis, Fuzhou University. C.P. Yang acknowledges partial support from the National Natural Science Foundation of China under Grant No. 11074062, the Natural Science Foundation of Zhejiang Province under Grant No. Y6100098, and the funds from Hangzhou Normal University.

[1] A. Rauschenbeutel, G. Nogues, S. Osnaghi, P. Bertet, M. Brune, J. M. Raimond, and S. Haroche, Phys. Rev. Lett. 83, 5166 (1999); Science 288, 2024 (2000).

[2] J. S. Bell, Physics 1, 195 (1965) .

[3] D. M. Greenberger, M. A. Horne, and A. Zeilinger, in Bell's Theorem, Quantum Theory, and Conceptions of the Universe, edited by M. Kafatos (Kluwer, Dordrecht, 1989); D. M. Greenberger, M. A. Horne, A. Shimony, and A. Zeilinger, Am. J. Phys. 58, 1131 (1990).

[4] A. K. Ekert, Phys. Rev. Lett. 67, 661 (1991).

[5] M. Hillery, V. Buzek, and A. Berthiaume, Phys. Rev. A 59, 1829 (1999).

[6] H. J. Briegel and R. Raussendorf, Phys. Rev. Lett. 86, 910 (2001); R. Raussendorf and H. J. Briegel, Phys. Rev. Lett. 86, 5188 (2001).

[7] T. Tanamoto, Y.X. Liu, S. Fujita, X. Hu, and F. Nori, Phys. Rev. Lett. 97, 230501 (2006); J.Q. You, X.-B. Wang, T. Tanamoto, and F. Nori, Phys. Rev. A 75, 052319 (2007); T. Tanamoto, Y.X. Liu, X. Hu, and F. Nori, Phys. Rev. Lett. 102, 100501 (2009).

[8] J. I. Cirac, P. Zoller, H. J. Kimble, and H. Mabuchi, Phys. Rev. Lett. 78, 3221 (1997); S. J. van Enk, J. I. Cirac, and P. Zoller, Phys. Rev. Lett. 78, 4293 (1997).

[9] A. Serafini, S. Mancini, and S. Bose, Phys. Rev. Lett. 96, 010503 (2006); Z. Q. Yin and F. L. Li, Phys. Rev. A 75, 012324 (2007); Z. B. Yang, H. Z. Wu, W. J. Su, and S. B. Zheng, Phys. Rev. A 80, 012305 (2009).

[10] S. B. Zheng, Appl. Phys. Lett. 94, 154101 (2009); S. B. Zheng, Chin. Phys. B19, 064204 (2010).

[11] T. J. Osborne and M. A. Nielsen, Quant. Inf. Process. 7, 45 (2002); Phys. Rev. A 66, 032110 (2002).

[12] A. Osterloh, L. Amico, G. Falci, and R. Fazio, Nature 416, 608 (2002). 
[13] G. Vidal, J. I. Latorre, E. Rico, and A. Kitaev, Phys. Rev. Lett. 90, 227902 (2003).

[14] M. J. Hartmann, F. G. S. L. Brandão, and M. B. Plenio, Phys. Rev. Lett. 99, 160501 (2007); Laser \& Photon. Rev. 2, 527 (2008).

[15] A. Imamoglu, D. D. Awschalom, G. Burkard, D. P. DiVincenzo, D. Loss, M. Sherwin, and A. Small, Phys. Rev. Lett. 83, 4204 (1999); A. Miranowicz, S. K. Özdemir, Y. X. Liu, M. Koashi, N. Imoto, and Y. Hirayama, Phys. Rev. A 65, 062321 (2002).

[16] L. Zhou, Z. R. Gong, Y. X. Liu, C. P. Sun, and F. Nori, Phys. Rev. Lett. 101, 100501 (2008); L. Zhou, H. Dong, Y. X. Liu, C. P. Sun, and F. Nori, Phys. Rev. A 78, 063827 (2008); L. Zhou, S. Yang, Y. X. Liu, C. P. Sun, and F. Nori, Phys. Rev. A 80, 062109 (2009); J. Q. Liao, Z. R. Gong, L. Zhou, Y. X. Liu, C. P. Sun, and F. Nori, Phys. Rev. A 81, 042304 (2010).

[17] S. B. Zheng and G. C. Guo, Phys. Rev. Lett. 85, 2392 (2000).

[18] D. W. Vernooy, A. Furusawa, N. P. Georgiades, V. S. Ilchenko, and H. J. Kimble, Phys. Rev. A 57, R2293 (1998).

[19] S. M. Spillane, T. J. Kippenberg, K. J. Vahala, K. W. Goh, E. Wilcut, and H. J. Kimble, Phys. Rev. A 71, 013817 (2005).

[20] S. M. Spillane, T. J. Kippenberg, O. J. Painter, and K. J. Vahala, Phys. Rev. Lett. 91, 043902 (2003).

[21] K. J. Gordon, V. Fernandez, P. D. Townsend, G. S. Buller, IEEE J. Quant. Electron. 40, 900 (2004).

[22] F. Dimer, B. Estienne, A. S. Parkins, and H. J. Carmichael, Phys. Rev. A 75, 013804 (2007). 